\title{
Etude sur les mots-dièse
}

\author{
Jackiewicz, Agata \& Vidak, Marko \\ STIH, Université Paris-Sorbonne \\ Agata.Jackiewicz@paris-sorbonne.fr, marko.vidak@yahoo.fr
}

\section{Introduction}

Notre communication présente une recherche linguistique portant sur les discours du réseau de microblogage Twitter. Il s'agit d'apporter une contribution à l'étude des nouvelles pratiques d'écriture sur les dispositifs numériques. L'objectif général visé est d'analyser, en linguiste, le microblogage (vu à la fois comme un dispositif technologique et un ensemble de pratiques sociales spécifiques) et ses influences sur les formes écrites et orales dans les discours contemporains. Nous nous intéresserons ici plus spécifiquement à l'outil d'indexation des messages sur le réseau Twitter qu'est le mot-dièse (ou hashtag HT).

Du fait du caractère récent du phénomène, peu d'études linguistiques traitent des pratiques discursives ayant cours sur Twitter. Nous nous appuierons essentiellement sur des travaux en analyse de discours (Longhi 2013, Longhi à paraître; Paveau 2011, 2013 ; Zapavigna 2011, 2012) et en TAL (Pak et Paroubek 2010 ; Davidov et al. 2010) traitant de problématiques connexes. Mais notre approche mobilise plusieurs cadres d'analyse, que nous expliciterons par la suite. En plus d'une analyse qualitative des faits de langue et du discours, notre apport entend tirer parti des analyses quantitatives systématisées sur de gros volumes de données, permettant d'attirer l'attention sur des faits récurrents et statistiquement significatifs.

Cette étude qualitative et quantitative a été menée sur un corpus de plusieurs milliers de tweets, émis en rapport avec le débat sur le mariage pour tous (corpus désigné par le sigle MPT). La collecte des observables a été réalisée à partir d'un ensemble de mots-dièse et de mots clés les plus caractéristiques (mariage homosexuel, gay, homo...). Le corpus MPT a été élaboré pour les besoins d'une première étude (Jackiewicz et Vidak, 2013) qui interroge les discours de Twitter selon plusieurs axes. Le travail ici présenté approfondit et problématise la question des mots-dièse, outils polymorphes et polyvalents, encore très peu analysés pour eux-mêmes.

Nous allons tout d'abord décrire le réseau Twitter et ses caractéristiques générales et discursives pour ensuite nous focaliser principalement sur l'étude linguistique du mot-dièse : les caractéristiques formelles, les propriétés sémantiques et pragmatiques, la modalisation du discours et la subjectivité, les fonctions linguistiques et sociales de cet indice spécifique au microblogage.

\section{Twitter et ses discours}

\subsection{Le réseau Twitter}

Twitter est un service de microblogage, créé en 2006 par Jack Dorsey et géré par l'entreprise Twitter Inc. Il permet d'envoyer de brefs messages, appelés tweets («gazouillis »), sur internet, par messagerie instantanée ou par SMS. A l'origine, Twitter a été pensé pour donner la possibilité à ses utilisateurs de raconter ce qu'ils font au moment où ils le font («What are you doing? ») et d'échanger des informations («What's happening?»). Aujourd'hui, Twitter invite tout simplement à composer un nouveau tweet («Compose new Tweet... »). Twitter est utilisé comme plateforme de réseau social permettant de relier et de fédérer des communautés de personnes au sens très large.

Tout utilisateur de Twitter peut publier des messages qui peuvent potentiellement être lus et/ou repris (cités, « retweetés ») par tout autre utilisateur. N'importe qui peut accéder au réseau et lire les messages 
qui ne sont pas protégés. Les membres du réseau peuvent s'abonner aux publications de n'importe quel autre utilisateur, devenir des followers (suiveurs). Ils voient alors s'afficher sur leur page d'accueil les messages postés par les personnes aux comptes desquelles ils sont abonnés.

Le nombre de messages postés sur ce réseau spécifique augmente en permanence. Pour un seul événement politique majeur aux États-Unis en 2012, le pic du nombre de messages envoyés par minute a dépassé trois cents milles unités. Dans le corpus MPT, le plus grand nombre de tweets récoltés dans une seule journée avoisine les deux cents milles (le 13 janvier, jour d'une manifestation contre la loi sur le mariage pour tous).

\subsection{Les tweets}

Un tweet est un message composé de 140 caractères au maximum. Il peut contenir différentes formes langagières : du texte simple, des mots-dièse (suites de caractères contigus précédés du croisillon \#, qui indexent le message), des pseudos (précédés de @, dispositif d'adressage), des liens URL vers des contenus extérieurs sur le Web, des émoticônes, des sigles qui renvoient à différentes fonctionnalités (RT : retweet, manière de signifier que le message qui suit est repris à quelqu'un d'autre). Ces formes sont combinables entre elles selon différentes logiques. De plus, des contenus multimédia peuvent y être adjoints (images, contenus vidéo, aperçus de pages Web et liens...). Pour reprendre les mots de Paveau (2012), «le tweet est une production plurisémiotique complexe, fortement contextualisée dans un écosystème technodiscursif. »

Certains tweets contiennent uniquement du texte rédigé de manière relativement conventionnelle, en respectant les normes de la langue écrite (quant à la morphologie et la syntaxe, notamment) et qui sont $a$ priori interprétables par tout un chacun (1). D'autres au contraire sont formés d'éléments composites en rupture avec les règles d'écriture habituelles (2). La part proprement textuelle peut d'ailleurs être réduite ou inexistante. Quand il est question d'exprimer un raisonnement plus complexe, le recours à des symboles $(+, \&,->,>,=)$ et à des formes nominales est une solution permettant de «tenir » dans les 140 caractères réglementaires $(3)$.

1) [OFFRE D'EMPLOI] L'Elysée cherche 1 latiniste et 1 linguiste maitrisant le français médiéval, pour recevoir les opposants au mariage gay.

2) C'est pas \#jolijoli... http://t.co/g0j5odjD \#prendstoipourleMJS et \#balance! http://t.co/On5Aj7cp @.JeudyBruno \#mariagegay

3) \#mariagegay -> \#adoption pour tous -> \#PMA pour les lesbiennes -> \#MerePorteuse pour les gays. Le debat, il est là. L'enfant $=$ pas 1 droit

La forme courte des messages de Twitter appelle une réelle concision de l'écriture. Celle-ci passe essentiellement par trois types de procédés : l'emploi de formes abrégées (symboles, abréviations, agglutinations, sigles, ellipse...), les emprunts dialogiques (détournements...) chargés de contenus implicites et l'usage de liens qui renvoient vers des documents extérieurs (4-6). Notons que l'insertion d'éléments cliquables tels que mots-dièse, liens, adresses d'interlocuteurs, conduit à une délinéarisation de l'énoncé, susceptible de différents modes de lecture.

4) Pr la PMA, le public n'a p-e pas atteint ce stade Françoise Heritier \#directAN \#mariagepourtous

5) Slogan : "Taubira, t'es foutue. Les familles sont dans la rue." \#ManifPourTous Vous n'avez pas le monopole de la famille. \#PointGiscard

6) "Et il est où ton papa ? Et elle est où ta maman ?" Jacques Martin http://t.co/ynJt73Kn \#Manifpourtous

Les usages et les registres de langue sont très diversifiés, étant donnée la généralisation de ce dispositif de microblogage, présent aujourd'hui dans quasiment toutes les sphères de la vie sociale, culturelle, institutionnelle et privée. A la pratique d'une langue lisse, normée et neutre, par exemple sur le service twitter de Radio France, s'opposent les discours privés, surchargés émotionnellement, proches des SMS, 
souvent transposés et raccourcis à partir des formes sonores de la langue orale (7). Quand il s'agit d'échanges entre personnes, même dans un cadre institutionnel, le style se rapproche fréquemment des bavardages oraux habituels (8).

7) ca mgave trp le truc sur le mariage homo,on est qui pour les jugés?personne. ils font ce qu'ils veulent, on choisit pas de qui on tombe amrx

8) le père godelier est intéressant mais il aurait mieux fait de préparer un papier, il part en couilles :) \#directan \#mariagepourtous

On trouvera chez Longhi (2013, à paraître) des analyses fines sur les caractéristiques des tweets politiques et des tweets institutionnels, montrant notamment l'influence des formes brèves sur le discours traditionnel, ainsi que le processus de l'hybridation de ces discours.

\section{Le mot-dièse : éléments de définition}

\subsection{Définition et principales caractéristiques}

Le mot-dièse est un marqueur de métadonnées couramment utilisé sur internet où il permet de marquer un contenu avec un mot-clé. Le Journal Officiel définit le mot-dièse (équivalent anglais : hashtag, canadien : mot-clic) comme une « suite signifiante de caractères sans espace commençant par le signe \# (dièse), qui signale un sujet d'intérêt et est insérée dans un message par son rédacteur afin d'en faciliter le repérage ». Cette définition est complétée par deux notes : «En cliquant sur un mot-dièse, le lecteur a accès à l'ensemble des messages qui le contiennent. L'usage du mot-dièse est particulièrement répandu dans les réseaux sociaux fonctionnant par minimessages ». (JORF n0019 du 23 janvier 2013)

Autrement dit, l'utilisateur peut « indexer » ses publications, en insérant simplement un dièse devant un mot ou un groupe de mots de son choix, pour que le réseau Twitter (Facebook ou encore Google+) considère que sa publication n'est pas isolée, mais faisant partie d'une thématique ou d'un sujet sur lequel parlent d'autres personnes. Le mot-dièse inscrit ainsi le message dans un fil thématique. Un tweet peut ne pas contenir de mot-dièse, comme il peut en contenir plusieurs et être ainsi indexé dans plusieurs fils d'information, à côté de tous ceux qui contiennent ce même mot-dièse. Dans (9), les mots-dièse présents permettent de retrouver le tweet dans quatre fils d'information qui concernent respectivement : (i) le Parti socialiste (\#PS), (ii) la procréation médicalement assistée (\#PMA), (iii) le débat sur le mariage pour tous (\#mariagepourtous), et (iv) les débats à l'Assemblée Nationale (\#DirectAN).

\section{9) Le groupe \#PS acte le dépôt de l'amendement pour la \#PMA \#MariagePourTous \#DirectAN}

Le fait que le mot-dièse soit directement intégré au tweet fait éclater la séparation entre la structure et le contenu, caractéristique des langages classiques de structuration de données (XML...) où les balises sont cachées par les navigateurs et ne sont pas lisibles pour les utilisateurs. Toutefois, rien n'empêche que les utilisateurs placent le signe dièse devant tout texte, parfois même de manière redondante (10).

10) " \#Hollande, touche pas à mon Code Civil !" \#codecivil \#manifpourtous

Il est important de noter que l'usage du mot-dièse n'est ni encadré, ni normé. Il fait partie du processus même de rédaction. Les utilisateurs du réseau Twitter choisissent spontanément les éléments de leurs discours qu'ils souhaitent marquer de la sorte. Il n'existe pas d'intervention extérieure (humaine ou automatique) a posteriori comparable à celle d'un documentaliste, par exemple, qui contrôlerait leur usage ou les gérerait en les regroupant, en les classifiant. Wal (2007) parle à ce titre d'un «étiquetage collaboratif » du fait qu'il est librement utilisé par la communauté. Le réseau social peut reprendre ces HT ou ne pas les remarquer. D'ailleurs les mots-dièse les plus populaires du moment figurent sur la page d'accueil de chaque compte. Remarquons que le mot-dièse, forme native de Twitter, se pratique désormais en dehors de ce réseau, notamment sur des panneaux publicitaires. Il est intéressant d'observer ces usages pour en dégager les modes, les fonctionnements linguistiques et les finalités. 


\subsection{Principales fonctions}

La présence d'un mot-dièse dans un tweet le rend «investigable », au sens de Zappavigna (2011), ce qui augmente sa probabilité d'être retrouvé, lu et retwitté et de ce fait joue sur son impact potentiel. Le retweetage est à l'évidence une fonctionnalité caractéristique de ce réseau social et l'une des finalités de bon nombre d'utilisateurs lorsqu'ils composent un message.

On décrit habituellement les mots-dièse comme moyen d'indiquer le thème du message. Aux yeux de Davies (2010), les hashtags sont « une façon de catégoriser les messages de Twitter référant à un même sujet ». Le JO parle du « sujet d'intérêt ». Zappavigna (2011), quant à elle, va plus loin, en insistant sur la double fonction de la classification entendue au sens large: faciliter la recherche des tweets et rendre possible la fonction d'affiliation ( «affiliation diffuse » ambient affiliation). Il s'agit de la possibilité de fédérer autour de valeurs communes - pouvant se définir en termes positifs comme négatifs - de communautés humaines réelles ou virtuelles.

Pour Paveau (2011), le mot-dièse est une production technolangagière, au même titre que l'hyperlien (lien renvoyant à une page internet) ou le bouton de partage d'information. C'est un technomorphème, «car [il] permet par [un] clic de redocumenter un thème ou une information ». L'auteure propose de distinguer, dans une classification heuristique, plusieurs types de hashtags :

- balise d'identification pour LT [Live tweet : tweet posté sur le lieu d'un événement se produisant en direct] et redocumentation

- balise d'identification et de catégorisation (domaines thématiques, extra- ou intra-tweet)

- balise générique

- balise de modalisation : commentaire métadiscursif, instruction interprétative, marque expressive

- balise-phrase «sur-discursive»

- le hashtag ludique, méta-hashtag.

Cette typologie donne un aperçu de la diversité des fonctions possibles des mots-dièse. Pour éclairante qu'elle soit, elle présente néanmoins l'inconvénient de mélanger des critères d'ordre différent (forme/contenu ; fonctions très générales et très spécifiques). Il convient de noter également que le terme de balise est ambigu. Il pourrait laisser penser qu'il existe des balises spécifiques ou dédiées pour chaque type d'emploi, comme en XML par exemple. Or ici, il n'en est rien. C'est la sémantique du mot précédé par le symbole dièse (\#) et la nature de la relation qu'il entretient avec le contenu du tweet et son contexte d'émission qui permettent de comprendre sa fonction. Nous aborderons la question de l'ambiguité sémantique et fonctionnelle ainsi que celle de la complexité pragmatique des mots-dièse dans la suite de la communication. L'essentiel de notre propos sera de montrer que la dimension langagière du mot-dièse l'emporte largement sur son côté technique.

\section{Le fonctionnement linguistique du mot-dièse}

Les mots-dièse apparaissent comme des outils polymorphes et polyfonctionnels. Polymorphes, car leur forme linguistique n'est ni figée ni prédéfinie, en dehors du fait qu'il s'agit d'une suite de caractères contigus précédés d'un dièse. Polyfonctionnels, car ils permettent non seulement de classifier mais aussi de donner des indications métalinguistiques importantes, de véhiculer des opinions, etc. Nous nous proposons d'organiser nos observations selon les critères formels, sémantiques et pragmatiques.

\subsection{Les mots-dièse dans le corpus MPT}

Avant de procéder à l'analyse qualitative du fonctionnement des mots-dièse attestés dans notre corpus, donnons-en une première caractérisation quantitative. Des explorations ponctuelles, centrées sur certaines formes ou certains patrons, ont été réalisées avec l'outil TXM (Heiden et al. 2010)1.

Le corpus MPT initial compte 518563 tweets récoltés sur deux mois au moment des principaux débats (politiques et publics) autour de la loi sur le mariage pour tous (de décembre 2012 à février 2013). 
Dans l'ensemble des 518563 tweets, 64\% contiennent des mots-dièse. Un tweet doté de mot-dièse en comprend entre 1 et 9 . Sur 140 caractères du tweet, les mots-dièse en occupent 19 en moyenne et peuvent aller jusqu'à la couverture totale du message. Ces informations donnent la mesure de l'importance numérique des mots-dièse dans le corpus étudié et invitent par là même à une étude approfondie de leur fonctionnement au sein des messages et plus largement dans l'univers du microblogage. Le fait qu'un tweet comprenne dans un grand nombre de cas plusieurs mots-dièse est une donnée d'observation importante qu'il conviendra d'étudier linguistiquement.

On atteste dans le corpus MPT 20231 formes différentes de mots-dièse. Toutefois près de la moitié d'entre elles n'y sont présentes qu'une seule fois. Seulement 35 mots-dièse apparaissent dans plus de 1000 tweets ; 345 ont une fréquence supérieure à 100. Si l'emploi des mots-dièse tend à en diversifier les formes, peu d'entre eux sont repris et créent de véritables fils d'information.

Thématiquement, à ce stade du débat, la problématique du mariage et plus largement de l'homosexualité est centrale dans le corpus, avec quelques HT vedettes très fréquents et un grand nombre de HT de fréquence faible. Parmi les premiers, notons \#mariagepourtous (101121), \#MariagePourTous (48628), \#Mariagepourtous (8243), \#mariagegay (6448), \#LGBT (1679). Les mots-dièse le plus souvent employés par les opposants au projet de loi en question - en référence dans un premier temps aux événements de contestation, puis au collectif qui en est né- sont \#manifpourtous (79775), \#ManifPourTous (28405), \#Manifpourtous (7505).

Il est à remarquer que les mots-dièse ayant trait à la filiation sont relativement peu nombreux et peu représentés dans les tweets analysés (\#PMA 3098, \#GPA 1179, \#adoption 493, \#homoparentalité 273...). Le déplacement du débat sur ce terrain s'est opéré progressivement, et correspond à une période non couverte par le corpus.

Il convient enfin souligner la présence significative des HT qui relèvent de la politique et des médias. La sphère de la politique est présente par le biais des mots tels que \#Hollande, \#PS, \#UMP, \#Taubira, \#Boutin, \#Delanoё, \#FN.... Certains noms propres se retrouvent dans des créations subjectivées (\#commelePSorganiseunflop, \#boutinade...). Ce point sera développé dans la section 5.2. L’implication et l'influence des médias dans le débat sur le MPT peuvent être mesurées grâce aux fréquences des HT tels que \#ONPC, \#Zemmour, \#BFMTV, \#AFP, \#TF1, \#France2, \#itele, \#cdanslair, \#MotsCroisés.

Précisons enfin qu'au stade actuel notre analyse se fonde uniquement sur le contenu du message. Elle ne tient pas compte de l'identité de l'auteur du tweet. Etant donné qu'une personne physique peut avoir plusieurs identités numériques sur ce réseau (ce qui peut conduire à des cas de dissociation énonciative au sens de Longhi (2013)), nous ne sommes pas en mesure d'intégrer ce paramètre dans notre démarche actuelle.

\subsection{La morpho-syntaxe du mot-dièse}

\subsubsection{Morphologie : les formes et les usages}

Du côté de sa structure morphologique, le mot-dièse est constitué du signe «\#» suivi de caractères alphanumériques contigus, formant une ou plusieurs unité de sens. Formellement, on peut ainsi distinguer :

- des lexèmes ou équivalents employés seuls: (i) des noms et des adjectifs essentiellement (\#stupide, \#ecoeurée, \#adoption, \#intolérance...), (ii) des interjections, des onomatopées (\#ah!, \#aheum, \#bah, \#Pffffff...),

- des sigles, avec ou sans référence externe : (i) institutionnalisés : \#UMP, \#PS, (ii) consacrés par des usages dans des univers particuliers : médias ( \#ONPC = titre de l'émission " On n'est pas couché »), débats à l'Assemblée Nationale (\#AN ou \#directAN, \#QAG 'question au gouvernement à l'AN'), emprunts intégrés à la langue courante (\#cqfd), (iii) des créations plus 
libres et intuitives (\#VTCN $=$ « vous trouvez cela normal », \#FF = «follow Friday », \#WTF = « what the fuck »),

- des dates, des horaires (\#17janvier, \#13h15, \#13Janvier2013, \#1hdumat ...),

- des syntagmes, des propositions et même des phrases entières: syntagmes (\#capricedestar, \#MariageGay), agglutinations de formes nominales (\#UnHommeUneFemme, \#UnPapaUneMaman, \#lperelmere), formes verbales (\#jemeurs), phrases complètes (\#jedisoui, \#mariezvouscommevousvoulez, \#rienafoutredevotrevie...).

Les verbes et les mots grammaticaux (prépositions, articles...) sont rares. Ils peuvent toutefois apparaître dans des enchaînements de mots-dièse formant des unités de sens ([\#HUMOUR, \#PAS, \#TRES, \#GAY] ${ }^{2}$; [\#nier, \#succès, \#manifpourtous ]).

Aux côtés des expressions obéissant à des règles de grammaire classiques, on rencontre des chaînes qui répondent à d'autres principes de formation. Des formes phonétiques, courtes, denses et transcrivant l'oral sont courantes (\#onlafè, \#WeshPovType) (voir 4.3), tout comme des abréviations de toutes sortes (pkoi, sf que, gvt, cple, ns/vs, $H / F, \&,+, 1,2 \ldots$ ). L'emphase et l'expressivité peuvent passer par la répétition de certaines lettres (\#ENOOOOORME, \#MerciBizouxxx, \#loooooooooooove, \#cooontre).

Des créations diverses, issues notamment du détournement polémique de mots-dièse devenus populaires, sont formées en préservant la construction ou la phonétique des expressions initiales. Ainsi \#ManifPourTous a donné également \#ManifPourTasses, \#ManifPourTouffe, \#ManifPourTourette, \#manifPloufPlouf, \#manifpartouze...

De nombreuses formes sont consacrées par l'usage. Il peut s'agir du sigle correspondant au titre d'une émission de télévision $(\# O N P C=$ «On n'est pas couché»), de l'expression qui s'est figée grâce à l'utilisation du hashtag (ex: \#mariagepourtous, \#unpapaunemaman), du nom d'une personnalité en vue (ex: \#ChTaubira ou \#Taubira = Christiane Taubira, ministre de la justice ayant porté la loi sur le mariage pour tous), de la désignation d'un événement «\#manif16décembre» (manifestation contre la loi en question, organisée le 16 décembre 2012).

11) RT@LToutes: Les photos LToutes de la \#manif16dec pour le \#mariagepourTous sont ici! http://t.co/2CNNtJgj

Les tweets qui comprennent des mots-dièse composés de plusieurs unités de sens posent problème de lisibilité. L'emploi de majuscule en initiale de chaque lexème permet une lecture plus aisée (\#mariagepourtous vs \#MariagePourTous). Toutefois, dans de nombreux mots-dièse les majuscules sont placées de manière aléatoire ou selon d'autres principes (\#champsDeMars, \#BomBaMerdes..).

Notons aussi que de nombreux procédés morphologiques se concurrencent en produisant plusieurs signifiants pour un même référent (\#ChTaubira, \#Taubira, \#ChristianeTaubira). Dans Twitter, pour le moment, chacune de ces formes donnera lieu à un fil thématique différent. Pour donner une idée de la créativité des twittos (auteurs de messages), dans le corpus MPT le mot mariage figure dans près de 300 formes différentes de mot-dièse (\#MariageHétéro, \#MariagePourLesHétérosSeulement, \#MariageASauvegarder, \#MariagePD, \#mariageLGBT, \#MariageHomo, \#MariageAdoptionGay, \#MariagePasTropPourTous, \#mariagepourtousmemeàplusieurs, \#Mariageàtroispourtous, \#MariagePourTousOuPourPersonne...).

\subsubsection{Propriétés syntaxiques}

Nous nous sommes intéressés à l'intégration du mot-dièse dans le message et à ses rapports avec les autres composants du tweet. D'un point de vue formel, toutes les positions dans le message sont techniquement permises et effectivement exploitées: initiale, médiane ou finale. En première approximation, on note que les mots-dièse en position médiane sont plus fortement intégrés à l'énoncé du tweet que ceux qui occupent les positions périphériques. Les degrés d'intégration du mot-dièse dans le 
message observés dans le corpus vont de l'indépendance totale à l'intégration syntaxique complète. Les cas de figure les plus caractéristiques sont :

- Indépendance syntaxique (simple adjonction),

- Commentaire phrastique, s'apparentant à un adverbe de phrase ou d'énoncé,

- Message composé exclusivement de mots-dièse, sans rapports syntaxiques apparents (juxtaposition allant jusqu'au style télégraphique),

- Prédominance des mots-dièse : on commence à apercevoir la structure syntaxique canonique,

- Un énoncé dans l'énoncé, une double énonciation,

- Intégration syntaxique totale (le mot-dièse remplit une fonction syntaxique).

Lorsqu'il est adjoint à l'énoncé, c'est-à-dire non intégré à la structure syntaxique de l'énoncé, le motdièse peut constituer un marqueur à valeur d'index pour permettre la classification et le repérage ultérieur du message à l'intérieur d'un fil thématique (\#Mariagepourtous). On peut noter que dans (12) le statut de \#manif16dec n'est pas univoque. Dans (13) l'emploi du mot-dièse s'apparente à celui d'un adverbe de phrase.

12) @christineboutin "Jésus avait deux Papa non?" \#Mariagepourtous \#manif16dec

13) On peut tu manifester contre ceux qui manifestent contre le mariage gay? \#franchement

Dans le corpus MPT, on observe une tendance à agglutiner les mots-dièse les uns aux autres, à les juxtaposer, quelle que soit la position choisie. Cela peut aller jusqu'à occuper l'intégralité du tweet, seuls le(s) mot(s)-dièse le composant alors (14). Cette configuration peut traduire un souci d'économie. Certains énumérations de HT peuvent s'interpréter comme de véritables propositions. Qui parle, à quel propos, exprimant quelle position, en citant les paroles de qui ? etc. Dans (15) on peut lire, nous semblet-il : « Jean-Luc Roméro, vous avez mon soutien total au sujet du mariage pour tous ».

14) \#MariagePourTous \#AdoptionPourTous \#PasDeProcreationMedical

15) @JeanLucRomero \#TotalSoutien \#MariagePourTous

Mais ce type d'emploi peut traduire aussi une forme de «bombardement» par des termes, pour renforcer la visibilité d'un thème (16), ou au contraire pour le tourner en dérision (17).

16) @SuperLizeM \#mariagepourtous \#Gay \#LGBT \#SiMonFilsEstGay \#GayFriendly \#Lesbians \#mariagepourtous \#love \#coeur

17) \#manifpourtous \#mariagepourtous \#sexPourTous \#polygamiePrTous \#PartouzePrTous \#DivorcePrTous \#AvocatsPrTous \#VieGachéePrTous \#DiablePTous

Il n'est pas rare que cette opération serve à bruiter intentionnellement l'espace médiatique (on pense aux « trolls », des personnes qui occupent l'espace et polluent les fils). Les tweets qui mélangent divers sujets d'actualité, sans lien apparent si ce n'est leur impact médiatique (\#MixeLesSujetsRelousde2013), en sont proches. Par exemple, dans 358 tweets \#Depardieu se trouve en cooccurrence avec \#mariagepourtous et avec d'autres mots-dièse. Il serait alors vain de chercher à reconstituer une structure propositionnelle supportant un message clair et univoque.

18) \#MariageGay, \#Depardieu, \#CalendrierMaya, \#Matrice

Dans (19), les différents mots-dièse (thématiques et contextuels) sont juxtaposés dans une énumération à d'autres mots du tweet. Les rapports entre les différents composants peuvent être reconstruits à partir de leur signification.

19) Hommes, femmes, \#nature, \#culture, \#sexe, \#genre: DÉCRYPTAGE http://t.co/VFxgP1c0 \#interview \#entretien \#mariagepourtous 
Un mot-dièse peut prendre la forme d'un énoncé complet. Cet emploi sert souvent à porter un acte de discours fort (20). Il lui est alors possible de nouer des relations rhétoriques avec le reste du message, par exemple pour former une séquence de nature argumentative (21).

20) \#JeComprendLes82PourcentsDeFrancaisQuiEnOnRienAfoutre

21) \#nonaumariagepourtous \#ouiàlafiliationpèremèreenfant

En position médiane, les mots-dièse participent en général à la structure prédicative de l'énoncé. L'on note une prédominance pour les fonctions équivalentes du GN ou du GAdj (sujet, attribut, complément du verbe, complément déterminatif...). Les cas où ils assument la fonction du verbe ou d'adverbe (25) étant beaucoup moins fréquents mais pas inexistants.

22) \#marinelepen dans \#Zemmour et \#naulleau a définitivement beaucoup de mal à prendre position ferme sur la question du \#mariagegay

23) Après une bonne \#manifpourtous un bon \#mcdopourtous

24) Entre 75 et 100000 rigolos dans les rues de Paris à la \#manifpourtous. Rebaptisons ça \#bidepourtous \#floppourtous ou \#gamellepourtous

25) On ne peut plus \#nier le \#succès de la \#manifpourtous \#manif13janv \#13janvier \#Hollande doit réfléchir \#maintenant !! http://t.co/v3JbssiB

26) C'est pas \#jolijoli... http://t.co/g0j5odjD \#prendstoipourleMJS et \#balance! http://t.co/On5Aj7cp @JeudyBruno \#mariagegay

On atteste même des configurations où des mots grammaticaux se trouvent pourvus de dièse, comme dans (27) où l'on remarque une tendance à l'emphase.

\section{7) \#manifpourtous "BANDE D'ENCULÉS" \#HUMOUR \#PAS \#TRES \#GAY \#OUPSSS \#MDR}

Différents types d'intégration syntaxique peuvent coexister dans le même message comme dans (28) : d'une part l'intégration syntaxique du mot-dièse, qui n'est autre que la proposition subordonnée hypothétique, et d'autre part l'adjonction à l'extérieur de la phrase, ce deuxième mot-dièse n'entretenant plus de rapports syntaxiques et sémantiques avec les autres constituants phrastiques et agissant en tant qu'indice de classification.

\section{8) \#SiMonFilsEstGay qui veut l'épouser ? \#MariagePourTous}

En somme, si les mots-dièse peuvent entrer dans des constructions syntaxiques canoniques, en règle générale ils tendent à s'en affranchir du fait de leur double statut (langagier et technique).

\subsection{Proximité avec l'oral}

Aux côtés des messages orthographiés selon les normes de l'écrit standard, l'on retrouve un grand nombre de messages calqués sur l'oralité, comme les textos (SMS) et les messages brefs des autres médias sociaux. La concision imposée a manifestement stimulé l'usage des abréviations, qu'elles soient empruntées ou tout simplement transcrites phonétiquement. D'autre part, la rapidité et la fréquence des échanges s'accompagnent d'une spontanéité de l'énonciation, comme c'est le cas à l'oral.

Des formes de transcription phonétique en sont probablement les premiers indices (\#FôLesExcuser, \#ralboldesegvt). Ces mots-dièse modalisent en plus le contenu du message, en y apportant une marque du niveau de langue et éventuellement une touche humoristique. Les abréviations côtoient les emprunts spontanés à l'anglais, phénomène courant dans ce type d'échanges.

29) Photo du@Le_Figaro sur \#manifpourtous \#justdoit \#onlafè \#13janvier http://t.co/KxYGj0Z8

Les simplifications (raccourcis) et les erreurs sont monnaie courante, présents jusque dans la construction des mots-dièse et dans leur emploi. 
30) \#CestLaLoose si tes pas pour le \#MariagePourTous

31) Slmt qqes centaines de pers à la manif pr le mariage homo selon \#FranceInfo \#manifpourtous de quel côté est la rue? \#pourquoionenparle?

32) Ya une meuf ki ma tuer c'matin ds ma classe pendan un deba sur le mariage homosexuel el a di "Dieu a créé Adam \& Eve pa Adam \& Steve" \#PTDR

Le corpus MPT atteste de la récurrence de mots-dièse construits sur le schéma : \#«çame $\mid c ̧ a m$ ' $+\mathrm{V}[+$ Attribut] » (par exemple : ça me dégoute/dérange/horripile/inquiète/énerve/écœure/tue/gave/gonfle/soule Irévolte/fout la gerbe, la honte, les nerfs... /donne la nausée, envie de rire... rend folle/malade... ce qui dans la forme complète donne \#çametdégoute, ...). Courantes à l'oral dans un registre familier voir grossier, ces expressions modalisent fortement le contenu du tweet en jouant sur son expressivité. Marquée par rapport à la norme écrite, rapidement reconnaissable et identifiable, cette écriture contribue également à la construction de l'image ou de l'éthos de l'auteur. En effet, le style et le niveau de langage peuvent indiquer l'âge ou l'origine sociale des locuteurs. Cela peut facilement induire chez le lecteur une adhésion ou un refus de lecture, selon qu'il s'y reconnaît ou non et constitue ainsi une marque multimodale (d'appartenance, de reconnaissance, de connivence...).

Notons au passage que les «nouveaux» emplois de juste ${ }^{3}$, également caractéristiques des échanges oraux, fonctionnent bien en tant que mots-dièse: \#JusteTellementVrai, \#JusteUnePtiteQuestion, \#justeunpeulents, \#JustePourInfo, \#JustePourLaFaireChier, \#justepourrire, \#JustePourVousDire...

Certaines marques d'oralité inscrivent clairement le message dans le cadre d'un échange, souvent vif et direct. L'emploi des interjections et des expressions qui servent à interpeller (discréditer, insulter...) directement l'autre en sont des moyens privilégiés.

33) \#MariagePourTous Y'a pas de raison. En plus ça laisse 2 places de plus de libres les gars ! \#BahOuais !

34)RT@GuestJb RT@NBeaup: Ok, donc j'ai recut une lettre du bahut pour aller manifester contre le mariage gay. \#groscons

Les interjections sont ici les marques de spontanéité, de proximité, de l'échange direct ou de la volonté d'insulter. Elles peuvent ici être considérées comme une marque de dialogisme, bien que, la plupart du temps, elles ne soient pas adressées à quelqu'un en particulier mais à une communauté large dans un souhait d'expressivité.

Les insultes en forme de mot-dièse sont particulièrement productives et récurrentes dans notre corpus, notamment sur le schéma \#bandedeX (" \#bandedeloosers », " \#bandedecons », " \#bandedefou », "\#BandeDeFdp», "\#BandeDeSousMerdes »...) ou \#allezvousVinfX ( \#Allezvousfairefoutre », " \#allezvousnoyerdansleaubénite », " \#Allezvouspendrebandedesalopes », " \#AllezVousPendre » ...).

35) Tout le \#monde me fait \#chier avec le \#référendum sur le \#mariagepourtous. Qu'on parle \#chômage, \# misère en \#france ! \#Merde !

36) Hommage a tous ces homophobes qui défilent dans Paris. \#bitedansvosculs \#manifpourtous

37) "Priez pour nous, on \#baise pour vous". Best \#slogan ever ! Ha ha ha ! http://t.co/daTStMMS

38) "On manifeste pour les enfants, pour sauvegarder la vraie famille." \#allezvousnoyerdansleaubénite \#imbéciles \#manifpourtous

L'adjonction du symbole «\#» peut également être une marque d'emphase, permettant d'insister ou d'attirer le regard. S'apparentent-elles alors à un marquage prosodique qui serait facilement reconnaissable à l'oral ? 


\subsection{Fonctions sémantiques et visées pragmatiques}

\subsubsection{Fonctions sémantiques}

D'un point de vue sémantique, les fonctions des mots-dièse peuvent être nombreuses: thématique, cadrative, médiative, modale, méta-linguistique, illocutoire... Selon la manière dont le tweet est rédigé (présence ou absence de structure syntaxique) et selon l'importance du contexte extralinguistique, ces fonctions seront plus ou moins faciles à reconnaître et à interpréter.

Un mot-dièse peut marquer le thème du message. En l'absence d'intégration syntaxique au message, en position frontale ou finale, leur fonctionnement peut être approché de celui des introducteurs de thématique tels que concernant $\mathrm{x}$, à propos de $\mathrm{x}$... Par exemple, il peut être question de l'adoption, du vote des étrangers, du code civil... (\#adoption, \#CodeCivil ...). De très nombreux tweets dans le corpus MPT contiennent le mot-dièse \#mariagepourtous qui rattache ce qui est dit au débat en question (39).

39) Que des élus de la république soient fiers de manifester pour priver une partie de la population d'un droit ça me dépasse ! \#MariagePourTous

Selon les cas, différents degrés de spécification sont obtenus par (i) la création d'un mot-dièse spécifique (\#VoteDesEtrangers, \#adoptionCoupleHomo, \#PMApourCoupleHomo, LesMeilleursCouacsDuPartiSocialiste2012...), (ii) par l'association de plusieurs HT thématiques au sein d'une liste. Ainsi, le sujet de l'adoption par des homosexuels pourra être spécifié par l'association de deux mots-dièse contigus \#adoption, \#homosexuels. La coexistence, même à distance à l'intérieur du tweet, de plusieurs mots-dièse thématiques amène le lecteur à rechercher des rapports sémantiques entre eux, qui peuvent être de différentes natures. Dans le corpus MPT, la forte cooccurrence de \#manifpourtous et de \#Hollande, par exemple, traduit le fait que François Hollande a été fortement pris pour cible par les opposants au mariage pour tous.

40) \#mariagepourtous \#civitas mieux vaut etre gay que cons sanguins !

41) \#Hollande doit se marier pour donner l'exemple. Le BON exemple serait d'épouser la mère de ses enfants évidemment. \#manifpourtous

42) \#LesMeilleursCouacsDuPartiSocialiste2012 : les élus \#PS qui ne signent pas l'amende \#PMA dans le texte de la loi \#mariagepourtous

Les mots-dièse médiatifs permettent d'indiquer la source de l'information ou le type d'information véhiculée (énonciateur, média, institution de référence...) : \#JTFrance2, \#arte, \#cdanslair, \#INSEE... Il peut s'agir d'un compte-rendu informatif ou d'une mention sensée légitimer ou crédibiliser le contenu transmis. Des tweets polémiques peuvent d'ailleurs contester les propos relatés par les sources en question.

43) \#motscroises A entendre les anti mariage homo, la famille tradi serait une sorte de perfection, un paradis. Ils plaisantent?

44) La Commission des lois de l'Assemblée Nationale adopte le projet de loi "Mariage pour tous" \#directAN

45) Pour le PACS contre le \#MariagePourTous ? \#OuEstLaLogique \#JTFrance2

Un mot-dièse peut désigner le destinataire du message, particulier ou collectif, notamment quand celui-ci joue un rôle important dans le débat (46).

46) \#hollande pourquoi ne pas faire voter le peuple ??? \#mariagepourtous le peuple dis non !!!

Les mots-dièse cadratifs indiquent où et quand se passe l'événement mentionné (un rassemblement, une manifestation...): \#lyon, \#denfertrochereau, \#27janvier, \#dimanche. Ils sont alors autant d'éléments référentiels, la plupart du temps de l'ordre spatio-temporel. Plus généralement ces mots-dièse servent à véhiculer le contexte (47-48). 
47) L'instrumentalisation de l'enfance c'est maintenant ! \#MariageHomo \#ManifPourTous \#13janvier @LaManifPourTous http://t.co/s1BCgulD

48) L'article 1 du projet de loi \#MariagePourTous est adopté par la commission des Lois après 8 heures de débat... \#DirectAN \#1hdumat \#clapdefin

Pour compenser l'absence de situation de communication partagée, certains mots-dièse fournissent les indications nécessaires à la compréhension du message, évitant l'erreur d'interprétation. Ce sont des marqueurs métalinguistiques qui peuvent en plus véhiculer l'information sur le genre et le registre de langage comme dans \#blague, \#joke, \#humour, \#euphemisme, \#ironie (49).

49) "Moi je suis pour le Mariage gay ...je n'ai pas envie de me mettre ces gens à dos" \#joke

La dimension modale des mots-dièse, remarquée dans les travaux de Zapavigna, est particulièrement présente dans le corpus MPT. En cause, le caractère très vif du débat social autour de cette question qui a réellement divisé la société française et continue à le faire après la promulgation de la loi. Différentes formes peuvent remplir ce rôle: marques modales simples (\#intox, \#excellent...), commentaires (\#lavieillesseestunnaufrage), noms communs dérivés de noms propres (\#Tartuffes, \#tartufferies...), questions rhétoriques (\#ElleEstOùTaLogique), requalifications... De nombreux mots-dièse réunissent au sein du même segment la cible et le jugement (\#HomophobieEstUnDélit...). Nous approfondirons ce point dans la section 5 .

50) Manif pour tous: Simone Veil a "salué les manifestants" contre le mariage gay http://t.co/Iy5k30zC \#lavieillesseestunnaufrage

51) La \#Manifpourtous c'est plutôt la \#ManifContreCertains !

\subsubsection{Visées pragmatiques}

Les mots-dièse servent à inscrire le message dans le flux des événements ordinaires ou extraordinaires de l'expérience humaine. Miroirs tendus aux destinataires, ils installent la complicité par l'appel à un vécu commun. Et relient non plus tant les tweets entre eux que les twitteurs. En même temps, ils ouvrent l'échange vers l'extérieur, en laissant la possibilité à une communauté large de réagir au message en question.

D’un point de vue pragmatique, l'emploi d'un mot-dièse peut servir des objectifs variés :

- Informer d'un évènement en temps réel ([\#DirectAn, \#mariagepourtous, \#QAG]) ou commenter en direct un évènement ([\#ONPC, \#mariagepourtous, \#décevant]),

- Inscrire ses publications dans des catégories ou thèmes de lecture (\#PMA, \#adoption),

- Essayer de transformer le thème de son message en « trending topic »,

- Recueillir des témoignages sur un sujet (\#parolesdenfantsdhomos),

- Constituer une communauté d'intérêt, maintenir le dialogue et le contact, rechercher la connivence (\#RemetsToiEnQuestionSi tu as participé-e à la \#manifpourtous),

- Participer à des actions collectives (\#TousLesJoursUnTweetPourFlorenceCassez; [\#onnelacherien, \#manifpourtous]),

- Construire son propre éthos, exprimer ses idées et ses positions personnelles,

- Dénoncer ou défendre un concept, une personnalité, une organisation (\#ToiAussiParleCommeBHL, \#Hollandedemission, \#TroisHommesEtUnBoutin, \#UMPanique...).

Cette liste des visées générales peut difficilement être close ou stabilisée. Par ailleurs, plusieurs objectifs peuvent être poursuivis simultanément. Longhi (2013) observe que les hashtags présents dans les tweets 
des politiques remplissent «une fonction stratégique, doublée d'une dimension performative puisque c'est aussi en créant des sujets que des candidats peuvent se rendre visibles ». L'utilisation de Twitter par un politique est censée montrer sa proximité avec le «peuple » (en réalité les électeurs potentiels) et sa capacité d'écoute (démocratie participative), tout en témoignant de sa modernité (capacité d'adaptation) et de son dynamisme.

\subsubsection{Quand un mot-dièse porte une « formule »}

Bon nombre d'énoncés sur Twitter fonctionnent de manière comparable aux « formules » ou aux «petites phrases », au sens de Krieg-Planque $(2009,2012)$. Rappelons qu'une « formule » est définie comme « un ensemble de formulations qui, du fait de leurs emplois à un moment donné et dans un espace public donné, cristallisent des enjeux politiques et sociaux que ces expressions contribuent dans le même temps à construire (Krieg Planque, 2009, p.7).

L'expression «mariage pour tous », devenue mot-dièse (\#mariagepourtous) correspond parfaitement à cette définition, comme on peut le montrer en examinant ses principales propriétés.

Commençons par le versant formel de l'expression «mariage pour tous ». En tant que mot dièse, elle est présente dans un tiers des tweets du corpus MPT, dans des variantes simples ou augmentées (\#MariageEtAdoptionPourTous, \#HétérosNousSoutenonsLeMariagePourTous, \#antimariagepourtous, \#nonaumariagepourtous ...). La stabilité de son signifiant, condition matérielle de reprise et de circulation, repose sur sa structure «X+pour+tous». Notre corpus compte plusieurs centaines d'expressions en -pourtous, attestées dans près de 270000 occurrences ( $52 \%$ des tweets). Le corpus témoigne en particulier de nombreuses formes détournées, à l'orientation argumentative plus ou moins transparente. L'esprit de la solidarité, du changement et du progrès social, dans la lignée de l'ouverture du mariage à tous les couples, peut être noté pour \#égalitépourtous, \#libertépourtous, \#amourpourtous, \#culturepourtous..., particulièrement quand les tweets les associent entre eux [\#BonneAnnée, \#TravailPourTous, \#MariagePourTous, \#LogementPourTous, \#DroitDeMourirDansLaDignité, \#DroitDeVoteDesEtrangers ]. Mais, dans beaucoup de cas, notamment quand on décèle un second degré, l'interprétation du mot-dièse devient plus délicate. Certaines expressions visent à critiquer ou à tourner en dérision le projet du mariage pour tous (\#miragepourtous), l'homosexualité, certaines mœurs ou pratiques (\#fumettepourtous, \#pédophiliepourtous, \#sodomiepourtous... ). D'autres ciblent le mariage tout court, en mettant en avant ses aspects les plus triviaux (\#repassagepourtous, \#vaissellepourtous, \#pelousepourtous, \#BelleMèrePourTous, \#laCamifpourtous). Plus largement, c'est l'ampleur du débat et l'effervescence sociale autour de cette question qui sont parfois pointées du doigt. Certains tweets contestent l'idée qu'une chose (droit, privilège...) puisse ou doive être partagée par tout le monde. Avec une ironie manifeste, les auteurs font alors semblant de réclamer pour tout le monde, ce qui en soi n'est pas désirable (\#suicideassistépourtous...). Notons que le mot-dièse le plus fréquent après \#mariagepourtous et \#manifpourtous - dans le paradigme \#Xpourtous - est \#floppourtous, mais ses attestations peuvent viser tant le mariage que la manif. De nombreux tweets contenant ce mot-dièse sont d'ailleurs ambigus.

L'expression linguistique «mariage pour tous » est née d'un débat social de grande ampleur. Elle est devenue une formule, à la faveur des échanges langagiers dans une situation sociolinguistique et historique bien identifiée. Désormais elle fait référence pour parler du mariage entre individus du même sexe. Elle a acquis un statut social. L'aspect polémique de son signifié est incontestable. Dans le corpus MPT, les nombreuses formes détournées à partir de l'expression originelle en témoignent très clairement. Aujourd'hui encore, malgré l'adoption de la loi, des enjeux touchant au mariage et plus largement à la famille sont vifs dans la société française et des questions essentielles à ce propos ne cessent d'être reposées.

Si l'une des conditions de l'existence d'une formule est sa circulation tous azimuts, aucun doute que l'expression «mariage pour tous » a trouvé une grande résonnance sur tous les médias. On note plus de 52 millions d'attestations sur le Web de «mariage pour tous » et 22 millions pour «manif pour tous » (pour une recherche faite le $1^{\text {er }}$ mars 2014). Par exemple, pour parler de la PMA, Le Figaro titre (le 24/02/2014) «Mariage pour tous : la justice butte sur l'adoption des enfants nés par PMA ». 


\subsubsection{Interaction avec le contexte}

Les discours de Twitter possèdent une dimension intertextuelle, qui est inhérente au dispositif même de microblogage, un dialogisme développé et un ancrage contextuel d'importance variable. Les références peuvent être interdiscursives ou extralinguistiques, explicites ou implicites.

Tout comme le lien vers les sites web et l'adressage, le mot-dièse participe à l'ouverture du contexte de lecture du tweet. Il s'interprète en fonction du contexte extra-linguistique, tant localement (selon la situation courante) que plus globalement, en jouant sur les connaissances partagées et la mémoire collective. On sait que la connivence s'installe notamment quand on fait appel à une culture commune (52). Des énoncés faisant parti du patrimoine culturel commun peuvent être repris et recyclés, par exemple défigés, aussi bien sur un mode plaisant qu'ironique (Quintin et al. 2011). Des particitations, au sens de Maingueneau (2004), retouchées ou non, peuvent parfaitement remplir ce rôle. Certaines d'entre elles seront employées comme mots-dièse (\#cestlaluttenuptiale, \#lavieillesseestunnaufrage...).

52) Slogan: "Taubira, t'es foutue. Les familles sont dans la rue." \#ManifPourTous Vous n'avez pas le monopole de la famille. \#PointGiscard

On constate que pour le débat sur le mariage pour tous, la présidence de François Hollande et de son gouvernement de gauche constituent des éléments saillants de cet arrière-plan discursif. On le voit notamment à travers l'usage de mots-dièse formés à partir de formules politiques forgées pour le candidat Hollande en 2012. D'une manière provocante, il s'agit d'aller sur son terrain, rappeler ses engagements, pointer ses manquements (\#MoiPrésident, \#LeChangementCestMaintenant, \#LaCultureJuridiqueCestPasMaintenant, LaDiversionCestMaintenant ...) $)^{4}$. Les éléments tels que \#flamby (et par association, \#danette) remontent plus loin dans la vie politique de F. Hollande. Ils servent à la fois à le désigner et le discréditer.

53) \#moipresident je ne respecte pas mes promesses de campagne sauf pour le \#mariagepourtous

54) y'avait pas des promesses pour l'emploi et contre la pauvreté aussi dans son programme? \#LOLhande \#MariagePourTous, \#ChomagePourBeaucoupTrop

55) Ce soir \#danette devra changer de \#pub, la \#france s est levée pour \#flamby ! \#manifpourtous \#lechangementcestmaintenant

Les cas de détournement immédiat de mots-dièse sont nombreux. On constate une forte créativité qui amène à reprendre (et à travestir) certains mots-dièse devenus populaires, pour polémiquer ou au contraire pour soutenir un message. Ainsi, \#ManifPourTous a donné entre autres \#manifPloufPlouf, \#UnHommeUneFemme a inspiré \#UnMixerUneCafetière, \#UnConUneConne etc. Comme nous venons de le voir dans la section précédente, l'expression Xpourtous, engendrée à partir de la formule mariage pour tous, a donné lieu à plusieurs centaines d'expressions différentes. Il est clair que l'interprétation de tous ces mots-dièse dérivés est indissociable de celle de leur modèle initial.

Le contexte socio-culturel est également fortement présent. Des références, directes ou indirectes, partagées par certains groupes d'âge, p.ex. à des styles de musique (\#GangnamStyle), à des centres d'intérêt ou des activités à la mode (\#FauxRaccordPourTous), des ouvrages et des films (\#ArmeeDeDumbledore, \#Inception). Des slogans hérités d'anciens combats (\#NoPasaran, \#faiteslamournonlaguerre, \#CEstLaLutteNuptiale...) sont réutilisés et adaptés.

\section{Mot-dièse en tant que marque de subjectivité}

Zappavigna (2011) affirme à juste titre que les mots-dièse entretiennent des rapports privilégiés avec les jugements d'évaluation. L'auteure souligne qu'ils représentent souvent la cible d'une évaluation, mais concède que rien n'empêche qu'ils expriment l'évaluation elle-même. Nous nous proposons maintenant d'explorer plus en détails les principaux emplois évaluatifs des mots-dièse. 


\subsection{Quelques procédés de modalisation}

La subjectivité et l'intersubjectivité des tweets répondent à la vocation même de l'outil, destiné à dire aux autres, en temps réel, spontanément et ouvertement, ce que l'on fait, pense ou ressent. L'émotion, l'emphase, l'humour en sont donc les moyens naturels. Sans pouvoir y accorder toute l'attention nécessaire, nous allons en décrire les principales caractéristiques.

Les tweets sont souvent porteurs d'opinions explicites et pleinement assumées. Les formes personnelles de nature déictique y sont très présentes, y compris sous forme de mots-dièse (formes en \#je: \#JeLeDisHautEtFort, \#commecavoussavezcequejepense, ...). Elles peuvent s'adresser directement à un interlocuteur (généralement collectif), sollicité, interpellé ou pris à parti (\#jededisçaatouslesbigots, \#occupetoidetesoignons, \#rentréchezvousetfaitespaschié...).

56) \#Jaiditoui au \#mariagepourtous à \#Angers! http://t.co/VADRdU9m

Dans le corpus MPT, on note la récurrence en tête du message et sous forme d'un mot-dièse d'expressions telles que: Ça me dégoute/dérange/horripile/inquiète/énerve/rend folle, malade... lécœure/tue/ gave/ gonfle/soule/ révoltelfout la gerbe, la honte, les nerfs .../donne la nausée, envie de rire...

Les champs sémantiques de la colère et de la honte y sont très présents (\#honteux, \#honte, \#HonteÀCetteManifRétrograde, \#hontesurlaFrance, \#, \#HonteDeMonPays, \#HonteAuGay...). Le calque de l'expression anglaise \#shameonyou est productif (\#hontesurvous, \#feusurlui, \#CaillouxSurvous, \#coeursurlui/elle/toi/vous, \#FuckSurVous, \#giflesurlui...).

57) C'est fou le nombre de tweets de jeunes \#gays en colère, tristes, blessés ... que je lis aujourd'hui \#Honte à la \#manifpourtous

La palette des moyens possibles est vaste et dépend de nombreux facteurs. Certains mots dièse ajoutent un jugement évaluatif au contenu qu'ils accompagnent $(58,59)$, d'autres réunissent la cible et l'opinion (\#fucklemariagegay, \#FuckLesHomophobes, \#HomophobieEstUnDélit, \#JapprouveLeMariageGay).

58) \#Delanoë demande 100.000 euros pour les dommages lors de la \#manifpourtous à la Préfecture de Police. \#WeshPovType

59) \#hollande a peur de se prendre une veste si \#referendum \#zérocourage \#mariagepourtous

Il est important de souligner que le rapport entre la cible d'évaluation et le jugement évaluatif est souvent à reconstituer, car la syntaxe ne le marque pas (comparer 60 et 61). Les mots-dièse modaux peuvent se trouver à tout endroit à l'intérieur du message (62).

60) " l'homosexualité c'est contre nature! " si c'etait contre nature, ça n'existerait pas, connard. \#desespéré \#mariagepourtous \#conneries.

61) Le monde change il parait mais ce dimanche est à vomir \#honte \#manifpourtous \#choquant

62) Ceux qui disent "Mariage pour Tous, on est tous égaux.." Non on est pas égaux, \#FeuSurVous t'es là grâce à l'homme \& la femme, n'oublie pas!

Des variantes morphologiques peuvent par exemple marquer la force d'une opposition (\#Cooooontre ; \#ContreA2000, \#CONTREEEEE, \#CONTRE+++++). Dans les messages à forte teneur subjective (appréciative, émotionnelle...) les marques modales sont souvent cumulées $(64,65)$.

63) "@JAE_3E: Eh ma TL, pr ou contre le mariage gay ?" \#CONTRE+++++!

64) Je suis tellement énervé par cette connerie humaine. Ces connards qui manifestent contre le mariage homosexuel \#FeuSurVous

65) @DamienFrc Les \#Homophobes sont les personnes contre l'égalité, contre le \#mariagepourtous. Voila la \#VraieHomophobie 
La diversité des réactions est également fonction de la diversité des cibles possibles. Autant dire, infinie. Les mots-dièse permettent par exemple de réagir à un discours (argumentaire) particulier (\#aucunargument, \#aucunelogique, \#Discoursdesplusintelligent_ENFIN, \#discoursridicule, \#discourshaineux...) ou à une campagne d'informations (\#bourragedecrane, \#confusion, \#cacophonie, \#intox).

66) Élisabeth Badinter dit "oui" au mariage pour tous.mp4: http://t.co/ld1v6zUm via @youtube \#Discoursdesplusintelligent ENFIN!! Ca

67) "On refuse de respecter le Naturel". Argument de la Droite à l'Assemblée, on croit rêver. \#HorsSujet \#MariagePourTous

68) La seule défense des anti-manifs sur Twitter c'est d'invoquer le nazisme et l'homophobie. \#confusion \#manifpourtous

Dans le débat sur le mariage pour tous, Tweeter a été utilisé pour diffuser des opinions, défendre des valeurs, se démarquer des autres au nom de ses valeurs (\#OnAPasLesMemesValeurs). Dans le corpus MPT, certains mots-dièse ont pour fonction d'énoncer (ou de rappeler) une norme, éventuellement assortie de préceptes d'ordre moral ou éducatif (69-71).

69) [\#UnPapaUneMaman, \#UnHommeUneFemme...]

70) [\#UnPapaUneMaman, \#OnNeMentPasAsesEnfants]

71) \#Maislemariagedoitetrecnstituerdunhommetunefemme

Au chapitre de la normativité, notons également le recours au second degré (\#40anstoujourspuceau) et l'exploitation humoristique des clichés, aussi bien pour plaisanter que pour tourner en dérision les idées du camp adverse (72-74).

72) Avec 2 papa PD plus d'enfants mal habillé \#mariagepourtous

73) " Un papa + une maitresse + une maman + un curé +12 enfants + une Renault Espace $=$ une famille normale \#manifpourtous "

74) Un papa + une maman + des pois chiches + du cumin + du citron $=$ \#manifhoumous \#manifpourtous \#mariagepourtous

\subsection{Désigner et interpeller l'autre}

Le débat sur le mariage pour tous s'est déroulé sur fond de clivages politiques, religieux, générationnels et culturels. Les opinions qui se sont exprimées dans ce contexte ont donc naturellement pris pour cible des individus et des institutions incarnant certaines visions de la société ou ayant adopté certaines positions sur le mariage, la famille ou la filiation.

Les joutes verbales sur Twitter, à l'aide de mots-dièse notamment, ont exploité des représentations stéréotypées associées aux différents participants du débat. Dans le corpus MPT, les deux communautés principalement visées sont celle des homosexuels et celle des catholiques. De nombreux mots-dièse ont été forgés pour l'occasion, notamment avec les radicaux «gay» et «catho ». Le procédé analogue a été exploité pour marquer le clivage politique entre la gauche et la droite (tableau 1).

Dans le corpus, peu d'entre eux servent à afficher l'appartenance de l'auteur du message (\#catholiques...). Certains explicitent la position défendue (\#legalisergaymariage). En règle générale, ces créations ont pour objet de disqualifier le camp adverse. 


\begin{tabular}{|c|c|}
\hline GAY & GAUCHE \\
\hline $\begin{array}{l}\text { \#Gay, \#GayEtFier, \#GayLib, \#ingaywetrust, } \\
\text { \#HeteroNousSoutenonsLeMariageGay, \#gaystapo, } \\
\text { \#gaymafia, \#lobbygay, \#gaypirates, } \\
\text { \#LesGayAuronsQuaBienCeTenir,\#antigay, } \\
\text { \#ManifAntiGay, \#unbongay, } \\
\text { \#TweetDeSuceurDeGays, \#SiMonFilsEstGay, } \\
\text { \#zlatanvousrendgay... }\end{array}$ & $\begin{array}{l}\text { \#gauche, \#chretiensdegauche, } \\
\text { \#DémocratieDeGauche, \#frontdegauche, } \\
\text { \#GaucheForte, \#GauchePourrie, } \\
\text { \#gauchiasses, \#gauchemolle, } \\
\text { \#BelleLaGÔche, \#Humourdegauche, } \\
\text { \#TesPasdeGaucheSi ... }\end{array}$ \\
\hline CATHO & DROITE \\
\hline $\begin{array}{l}\text { \#catho, \#catholiques, \#catholicisme... } \\
\text { \#CatholiquePourlaFamille, \#TeamCatho, } \\
\text { \#Cathosphère, \#cathostyle, \#cathomaispastrop, } \\
\text { \#CathoParade, \#cathopride, \#CathoRétroRéac, } \\
\text { \#cathocon, \#cathocharia, \#CathoAttardés, } \\
\text { \#HypocritesCathos, \#lobbycatholique, \#GrosCatho, } \\
\text { \#marreDesCathos, \#caholiquesdemescouilles, } \\
\text { \#maladieAlzheimergagnelecatho... }\end{array}$ & $\begin{array}{l}\text { \#droite, \#droitemoderne, \#droiteforte, } \\
\text { \#peuplededroite, \#droitepourlemariage, } \\
\text { \#ladroiteréac, \#droitehomophobe, } \\
\text { \#droitedécomplexée ... }\end{array}$ \\
\hline
\end{tabular}

Tableau 1 : Exemples de HT associant désignateurs et formes modales

De manière plus marginale, le débat à coup de stéréotypes n'a épargné ni les sportifs de haut niveau (75), ni les ouvriers en bâtiment (76)... Bon enfant pour certains, incisifs voire injurieux pour d'autres, les qualificatifs choisis ou crées par des twittos demandent à être analysés, car ils révèlent ce qui est spontanément mis en avant pour désigner, critiquer ou disqualifier l'autre. On constate, sans surprise, que certains attributs catégoriels sont choisis préférentiellement pour motiver ou supporter des jugements de valeur $(77,78)$.

75) Quand le mariage pour tous sera enfin en place, je suis sur que Zlatan va avoir des tonnes de demandes ... \#zlatanvousrendgay ...

76) \#lesmecsduchantier sont généralement contre le mariage gay

77) Jeunes \#LGBT, oubliez la \#manifpourtous - Vous n'êtes ni des malades, ni des erreurs de la nature, ni des pécheurs \#SoutienAuxJeunesLGBT

78) ATTENTION ATTENTION: avis de manif intemporelle de la droite \#DentiersetChapelets + \#CathoAttardés. \#MariagePourTous \#LaFranceAPeur

Dans la même logique, les noms des personnalités (notamment politiques) impliquées dans le débat MPT ont été exploités pour former des mots-dièse, à la fois référentiels et modaux. Là encore, différents cas de figure sont constatés, pouvant aller d'un inoffensif jeu de mot (79) à une insulte, blessante voire diffamatoire. Dans le corpus MPT, plusieurs dizaines de mots-dièse différents concernent François Hollande (dans près de 5000 tweets). Dans 700 attestations, nous trouvons des formes modifiées, telles que \#lafauteàHollande, \#HollandeEcouteTonPeuple, \#HollandeEnFumé, \#hollandeimpopulaire, \#HollandeSeMarie, \#Hollandouille, \#Hollandreou, \#HollandréouDernier, \#faisonsplierhollande, \#3MotsPourQualifierHollande, \#HollandeDémission, \#DehorsHollande, \#HollandeJeTeHais, \#hollandehitler, \#HollandeImposteur, \# hollandelediviseur, \#UneRésolutionPourHollande... De son côté, dans un registre souvent fort basique, Christine Boutins'est vue affublée de \#LaBoutinDeLaRaiePublique, \#CacaBoutin, \#boutinpauvreconne, \#DansTesDentsChristine, \#LaVieSelonChristine, \#ÄPoilChristine.

79) Christine Boutin et sa definition du mariage gay... C'est plus une boutade, c'est une \#boutinade ... Faut que ce soit en tendances. 


\section{Conclusion}

Notre travail entend apporter une contribution à la caractérisation des univers discursifs à support numérique. Dans cette optique, nous avons choisi de nous pencher sur les usages des mots-dièse.

Le mot-dièse est généralement présenté comme un moyen de classification des messages sur le réseau Twitter. Or, son fonctionnement discursif montre qu'il ne se résume pas à cette seule fonction, bien que celle-ci soit significative. De même, l'on ne peut se contenter de le qualifier de «trait technolangagier » sans décrire précisément comment ces deux composantes interagissent entre elles. Les mots-dièse font partie intégrante des structures productrices de sens, tout en offrant un moyen technique d'organiser et d'explorer les flux de messages ainsi produits. Enfin, les mots-dièse sont des témoins éloquents des usages de Twitter, culturellement et socialement marqués.

De par sa forme morphologique, il s'apparente à un élément multidimensionnel apte à encoder tant des éléments de sens simples que des contenus discursifs sémantiquement et argumentativement riches. Du point de vue syntaxique, l'on constate différents degrés d'intégration dans le contenu propositionnel. Cela va d'une indépendance totale par rapport au corps du tweet, jusqu'à l'intégration syntaxique complète où les mots-dièse assument les rôles argumentaux classiques. Dans la majorité des cas, une part d'interprétation est laissée au lecteur. L'usage et la confection du mot-dièse sur Twitter étant peu contraints, nous constatons une évolution sémantico-pragmatique très rapide, qui va dans le sens à la fois d'enrichissement de son rôle à l'intérieur du message que de multiplication de ses fonctions au sein du dispositif. A l'évidence, l'examen du corpus MPT montre que les utilisateurs de Twitter se sont emparés du mot-dièse de manière très libre, pour en faire un outil polyvalent et un véritable support de créativité.

En constante évolution, tout comme la communication sur les réseaux sociaux, le mot-dièse est encore peu exploré et surtout sous-estimé pour son potentiel linguistique et pragmatique. Des recherches s'appuyant sur les acquis de notre recherche, menées sur d'autres corpus et en rapport avec d'autres pratiques de communication, permettraient de compléter cette première étude en forme d'esquisse.

Les discours de Twitter sont de plus en plus constitués en corpus d'études pour des applications informatiques. Il est donc essentiel de pouvoir disposer d'analyses linguistiques préalables pouvant servir de base à des traitements automatiques finalisés. Notre travail, de par son caractère descriptif et systématique, aide à mieux cerner la nature de ce type d'observables, trop souvent encore enfermés dans des hypothèses simplificatrices, postulant l'unicité thématique et/ou axiologique des messages de Twitter.

Enfin, nous espérons avoir montré qu'un matériau discursif issu du Twitter, interrogé avec des outils linguistiques et informatiques, constitue une ressource précieuse pour des recherches à visée sociétale. Nous pensons par exemple qu'une perspective linguistique sur les discours produits et véhiculés par des réseaux sociaux devrait aider à expliciter la façon dont les gens se servent du langage pour construire de nouvelles lignes sociales autour des systèmes de valeurs en mutation ou en émergence.

\section{Références bibliographiques}

Davidov, D., Tsur, O., Rappoport, A. (2010). Enhanced Sentiment Learning Using Twitter Hashtgs and Smiley. Coling 2010.

Davies, B. (2010). The Language of Twitter Linguistic innovation and character limitation in short messaging. Accessible en ligne sur http://lewisdenby.files.wordpress.com/2010/06/the-language-of-twitter-linguisticinnovation-and-character-limitation-in-short-messaging.pdf. Consulté le 14.03.2014.

Heiden, S., Magué, J.-Ph., Pincemin, B. (2010). TXM : Une plateforme logicielle open-source pour la textométrie conception et développement. JADT 2010 : 10th International Conference on the Statistical Analysis of Textual Data, Rome, Italie.

Jackiewicz, A., Vidak, M. (2013). Le mariage pour tous en 140 caractères du tweet. Colloque international Français parlé dans les médias. Montpellier. 
Journal Officiel, (2013). JORF $\mathrm{n}^{\circ} 0019$ du 23 janvier 2013 page 1515, texte $\mathrm{n}^{\circ} 103$ : «Vocabulaire des télécommunications et de l'informatique ».

Krieg-Planque, A. (2009). La notion de "formule » en analyse du discours. Cadre théorique et méthodologique. Besançon : Presses Universitaires de Franche-Comté

Krieg-Planque, A. (2012). Analyser les discours institutionnels. Paris : Armand Colin.

Longhi, J. (2013). Essai de caractérisation du tweet politique ». Information grammaticale, 136.

Longhi, J. (à paraître). L'hybridation à l'épreuve du numérique : renouvellement et reconfiguration de la parole institutionnelle. J. Longhi et G.E. Sarfati (éds.), Les discours institutionnels en confrontation. L'Harmattan, Espaces Discursifs.

Maingueneau, D. (2004). Hyperénonciateur et 'particitation' ». Langages, 156, 111-126.

Mischaud, E. (2007). Twitter: Expressions of the Whole Self. An investigation into user appropriation of a web-based communications platform. London: Media@lse. Accessible en ligne sur http://wiredpen.files.wordpress.com/2009/05/mischaud final.pdf. Consulté le 14.03.2014.

Pak, A., Paroubek, P. (2010). Le microblogage pour la microanalyse des sentiments et des opinions. TAL $51: 3$.

Paveau, M.-A. (2013). Hashtag. Carnet de recherche. Technologies discursives, Accessible en ligne sur http://technodiscours.hypotheses.org/488. Consulté le 30.06.2013.

Paveau, M.-A. (2011). Genre de discours et technologie discursive. Tweet, twittécriture et twittérature. Conscila « Modèles et théories du genre en confrontation », à paraître dans la revue Pratiques, 2013.

Quintin, E., Jackiewicz A., Roy T. (2011). Analyse d'énoncés politiques à fort impact et éléments pour leur détection automatique. J. Bres, A. Nowakowska, J.-M. Sarale, S. Sarrazin (coord.) Actes du colloque international Dialogisme : langue, discours (8-10 septembre 2010, Montpellier). Mis en ligne le 10 juillet 2011.

Wal, W.T. (2007). Folksonomy coinage and definition, Accessible en ligne sur http://www.vanderwal.net/folksonomy.html. Consulté le 14.03.2014.

Zappavigna, M. (2011). Ambient affiliation: A linguistic perspective on Twitter. New Media \& Society. Accessible en ligne sur http://williamwolff.org/wp-content/uploads/2013/09/zappavigna-twitter-ambient-2011.pdf. Consulté le 14.03.2014.

\footnotetext{
${ }^{1}$ Les auteurs remercient chaleureusement Aymen Elkhlifi pour son aide dans le maniement informatique du corpus.

${ }^{2}$ Les crochets indiquent des séries de mots-dièse attestés dans le même tweet.

${ }^{3}$ Gilles Siouffi, Agnès Steuckardt et Chantal Wionet, «Enquêter sur des diachronies courtes et récentes : problèmes et méthodes, d'après l'exemple des modalisateurs de l'énoncé ». Exposé oral, STIH GEHLF, Paris, 11 mai 2012.

${ }^{4}$ Longhi dans (2013, pp.26-27) discute et illustre les différentes reprises sur Twitter du slogan socialiste Le changement c'est maintenant.
} 